Für $H_{x} \rightarrow 0$ ergeben sich aus Gl. (64) die beiden Lösungen :

$\left(v_{x, 1}\right)_{+}^{2}=\frac{\varrho_{2}}{\varrho_{1}} \frac{\Delta p}{\Delta \varrho}+\frac{1}{8 \pi \varrho_{1}}\left[H_{y, 1} H_{y, 2}+\left(H_{y, 2}\right)^{2}\right]$.

Dies stimmt mit Gl. (50) überein und ist die Geschwindigkeit einer longitudinalen, magneto-hydrodynamischen Stoßwelle, die senkrecht zum Magnet- feld verläuft. Im Grenzübergang $(\Delta \rightarrow 0)$ kommt man zum gleichen Resultat wie in Gl. (51). Außerdem ergibt sich:

$$
\left(v_{x, 1}\right)_{-}^{2}=0 .
$$

Dieses ist die Geschwindigkeit einer transversalen, hydrodynamischen Stoßwelle, die, wie bekannt, den Wert Null hat.

\title{
Bemerkungen zur Born-Oppenheimerschen Näherung für die Behandlung von Stoßproblemen
}

\author{
Von Karl Wildermuth \\ Aus dem Max-Planck-Institut für Physik, Göttingen \\ (Z. Naturforschg. 8a, 284-292 [1953]; eingegangen am 10. Januar 1953)
}

\begin{abstract}
An Hand eines einfachen Beispiels wird die analytische Struktur des Oppenheimerschen Näherungsverfahrens untersucht. Dabei stellt sich heraus, daß man durch dieses Verfahren (in seiner ursprünglichen Formulierung) die Ununterscheidbarkeit gleicher Teilchen auch nicht näherungsweise berücksichtigen kann (analog zum Bornschen Verfahren). Es wird kurz diskutiert, wie man dieses Verfahren abändern muß, um diese Ununterscheidbarkeit berücksichtigen zu können. Zum Schluß wird noch in einer graphischen Darstellung der elastische Streuquerschnitt für dieses Beispiel in verschiedenen Näherungen zu Vergleichszwecken aufgezeichnet.
\end{abstract}

$\mathrm{F}$ ür viele Probleme in der Physik, Geophysik und Astrophysik ist es wichtig, quantitative Angaben über die Streuquerschnitte und Ionisationswirkungsquerschnitte für die Streuung von Elektronen an Atomen und Molekülen zu besitzen. Die gebräuchlichste Methode zur Berechnung dieser Wirkungsquerschnitte ist die Bornsche Näherungsmethode. Zur Anwendung dieses Verfahrens muß man die Wellenfunktion nach den Eigenfunktionen des gebundenen Elektrons entwickeln. Außerdem muß man die Wechselwirkung der Stoßelektronen mit den Streuatomen bzw. Molekülen als kleine Störung ansehen. Das bringt aber mit sich, daß man den Einfang der Stoßelektronen unter Ionisation von Hüllenelektronen der Atome bzw. Moleküle (Austauschstreuung) auch nicht näherungsweise berücksichtigen kann, da dazu die Wechselwirkung der Stoßelektronen mit dem Atom- bzw. Molekülrumpf nicht mehr als klein betrachtet werden darf ${ }^{1}$. Weiterhin ist die Bornsche Näherung bezüglich der Behandlung der Stoßelektronen und der gebundenen Elektronen unsymmetrisch. Konsequenterweise wird daher bei dieser Methode in der

${ }^{1}$ K. Wildermuth, Z. Physik 127, 92 [1949].

2 J. R. Oppenheimer, Physic. Rev. 32, 361 [1928].
Wellenfunktion 0-ter Näherung auch streng zwischen Stoßelektronen und gebundenen Elektronen unterschieden und man muß nachträglich die erhaltene Lösung symmetrisieren. Um die oben erwähnte Austauschstreuung und die Ununterscheidbarkeit der Elektronen wenigstens näherungsweise berücksichtigen zu können, hat $\mathrm{O}$ p penh eimer vorgeschlagen, die Eigenfunktion 0-ter Näherung symmetrisch bzw. antisymmetrisch in den Elektronenkoordinaten anzusetzen ${ }^{2,3}$. Dieses Näherungsverfahren wurde bis jetzt immer nur bis zur ersten Näherung durchgeführt, und bei der mathematischen Kompliziertheit der Probleme, auf die es angewandt wurde, war es auch nicht möglich zu sehen, wie weit es als erster Schritt eines konsequenten Näherungsverfahrens angesehen werden kann, wie z. B. das Bornsche Näherungsverfahren, das eine Entwicklung der Streuwellenfunktion nach dem Kopplungsparameter darstellt. Es ist daher von Interesse, an Hand eines einfachen Beispiels, das sich mathematisch verhältnismäßig leicht durchschauen läßt, diese Methode genauer zu untersuchen, um z. B. Aussagen über ihren Gültigkeits-

${ }^{3}$ D. R. Bates et al., Philos. Trans. Roy. Soc. [London], Ser. A 243, 93 [1950]. 
bereich zu gewinnen. Außerdem kann man an diesem Beispiel auch erkennen, wie man die Bornsche und Oppenheimersche Näherungsmethode miteinander verbinden muß, um möglichst gute Resultate für die verschiedenen Streuquerschnitte zu erhalten.

\section{Erläuterung und Untersuchung des Beispiels}

Als Beispiel für unsere Betrachtungen wählen wir folgendes eindimensionale Zweikörperproblem:

a) Die Wechselwirkungen der beiden Teilchen mit dem Ursprung werden durch $\delta$-funktionsartige Potentiale beschrieben.

b) Die Wechselwirkung zwischen den beiden Teilchen wird ebenfalls durch ein $\delta$-funktionsartiges Potential dargestellt.

c) Vor dem Stoß besitzt das Teilchen 1 den Impuls $K_{0}$ und das Teilchen 2 ist an den Ursprung gebunden.

Setzt man alle hier unwesentlichen Konstanten gleich 1, so wird dieses Modell durch folgende Schrödinger-Gleichung beschrieben:

$$
\begin{aligned}
& \left\{-\frac{\partial^{2}}{\partial x_{1}{ }^{2}}-\frac{\partial^{2}}{\partial x_{2}{ }^{2}}-2 B \delta\left(x_{1}\right)-2 B \delta\left(x_{2}\right)\right. \\
& \left.\quad+A \delta\left(x_{1}-x_{2}\right)\right\} \psi\left(x_{1} x_{2}\right)=E \psi\left(x_{1} x_{2}\right) .
\end{aligned}
$$

Vergleichen wir (1) mit der Schrödinger-Gleichung für die Streuung von einem Elektron am Wasserstoffatom, so entspricht $A \delta\left(x_{1}-x_{2}\right)$ der Wechselwirkung der beiden Elektronen untereinander, während $-2 B \delta\left(x_{1}\right)$ und $-2 B \delta\left(x_{2}\right)$ der Wechselwirkung der Elektronen mit dem Proton entsprechen.

Zur Lösung und mathematischen Untersuchung (1) ist es zweckmäßig, in den Impulsraum überzugehen $^{1}$. Z. B. lassen sich aus den Wellenfunktionen im Impulsraum viel besser die verschiedenen Wirkungsquerschnitte berechnen als aus den Wellenfunktionen im Ortsraum.

Setzt man

$$
\psi\left(x_{1} x_{2}\right)=\frac{1}{2 \pi} \int_{-\infty}^{+\infty} f\left(k_{1} k_{2}\right) e^{i\left(k_{1} x_{1} k_{2} x_{2}\right)} \mathrm{d} k_{1} \mathrm{~d} k_{2}
$$

in (1) ein ${ }^{4}$, so erhält man für die Fourier-Transformierte $f\left(k_{1} k_{2}\right)$ folgende Integralgleichung ${ }^{5}$ :

$$
\begin{aligned}
& \left(k_{1}^{2}+k_{2}^{2}-E\right) f\left(k_{1} k_{2}\right) \\
& \quad-\frac{B_{1}}{\pi} \int f\left(k_{1} k_{2}\right) \mathrm{d} k_{1}-\frac{B_{2}}{\pi} \int f\left(k_{1} k_{2}\right) \mathrm{d} k_{2} \\
& +\frac{A}{2 \pi} \int f\left(k_{1}-k, k_{2}+k\right) \mathrm{d} k=0, B_{1}=B_{2} .
\end{aligned}
$$

Da es uns im Hinblick auf die Bornsche und Oppenheimersche Näherungsmethode darauf ankommt, Lösungen von (3) in Abhängigkeit von der Wechselwirkung der Teilchen mit dem Ursprung zu untersuchen, können wir die gegenseitige Wechselwirkung der beiden Teilchen immer als klein ansehen, ohne dabei etwas prinzipiell Wichtiges zu vernachlässigen. Bei den weiteren Betrachtungen wird daher die gegenseitige Kopplung der beiden Teilchen immer nur in erster Näherung berücksichtigt.

Es soll nun zunächst in erster Näherung bezüglich der Kopplungskonstanten $A$ die Lösung von (1) hergeleitet werden, mit der nachher die Bornsche und die Oppenheimersche Näherung (bezüglich der Kopplungskonstanten $B$ ) verglichen wird. Dazu wird folgender Ansatz für die Wellenfunktion gemacht:

$$
f\left(k_{1} k_{2}\right)=f_{0}\left(k_{1} k_{2}\right)+f_{1}\left(k_{1} k_{2}\right)
$$

$$
\operatorname{mit}^{6} f_{0}\left(k_{1} k_{2}\right)=\{\underbrace{}_{\alpha \rightarrow 0} \delta\left(k_{1}-K_{0}\right)+\frac{K_{0}}{K_{0}-i B} \frac{B}{\pi} \frac{1}{k_{1}^{2}-K_{0}{ }^{2}-i \alpha}\} \frac{N}{k_{2}{ }^{2}+B^{2}}, N=\sqrt{\frac{2}{\pi}} B^{3} .
$$

$f_{0}\left(k_{1} k_{2}\right)$ stellt eine Lösung der Gl. (3) für $A=0$ dar, die beschreibt, daß von links her ein Teilchen 1 mit dem Impuls $K_{0}$ einfällt und am Ursprung gestreut wird und ein Teilchen 2 mit der Energie $-B^{2}$ am Ursprung gebunden ist ${ }^{1}$. Die Bindung des Teilchens 2 mit dem Ursprung wird durch die auf 1 normierte Eigenfunktion $N /\left(k_{2}{ }^{2}+B^{2}\right)$ beschrieben. Außer diesem Bindungszustand existiert kein weiterer Bindungszustand für die Teilchen 2 mit dem Ursprung. Die Wellenfunktion für den entsprechenden Bindungszustand des Teilchens 1 am Ursprung lautet $N /\left(k_{1}{ }^{2}+B^{2}\right)$.

${ }^{4}$ Der Faktor $1 / 2 \pi$ ist notwendig, damit die Wellenfunktion im Ortsraum richtig normiert ist, wenn sie im Impulsraum richtig normiert ist.
5 Die Unterscheidung zwischen $B_{1}$ und $B_{2}$ in (3) ist für die späteren Hinweise auf Gl. (3) zweckmäßig.

6 Fußnote s. nächste Seite. 
Zur Lösung von (3) entwickeln wir $f_{1}\left(k_{1} k_{2}\right)$ nach den Eigenfunktionen des verkürzten Hamilton-Operators (3), in $\operatorname{dem} A=0$ und $B_{2}=0$ gesetzt ist. Wir setzen also analog zur Bornschen Näherung an:

mit den Eigenfunktionen ${ }^{6}$

$$
f_{1}\left(k_{1} k_{2}\right)=\int\left(K_{1} \mid k_{1}\right) g\left(K_{1} k_{2}\right) \mathrm{d} K_{1}+\left(1_{B} \mid k_{1}\right) g\left(k_{2}\right)
$$

$$
\left(K_{1} \mid k_{1}\right)=\underset{\alpha \rightarrow 0}{\delta}\left(k_{1}-K_{1}\right)+\frac{\left|K_{1}\right|}{\left|K_{1}\right|-i B} \frac{B}{\pi} \frac{1}{k_{1}^{2}-K_{1}^{2}-i \alpha}, \quad\left(1_{B}\left|k_{1}\right|=\frac{N}{k_{1}^{2}+B^{2}} .\right.
$$

Die Eigenfunktionen $(5 \mathrm{~b})$ und $(5 \mathrm{c})$ sind dabei folgendermaßen normiert:

$$
\int\left(K_{1} \mid k_{1}\right)\left(K_{1}{ }^{\prime} \mid k_{1}\right) * \mathrm{~d} k_{1}=\delta\left(K_{1}-K_{1}{ }^{\prime}\right), \int\left(1_{B} \mid k_{1}\right)\left(1_{B} \mid k_{1}\right) * \mathrm{~d} k_{1}=1 .
$$

Setzt man (5a) in (3) ein und multipliziert mit $\left(K_{1} \mid k_{1}\right)^{*}$ bzw. $\left(1_{B} \mid k_{1}\right)^{*}$ durch und integriert über $k_{1}$, so erhält man folgende Integralgleichungen für $g\left(K_{1} k_{2}\right)$ und $g\left(k_{2}\right)$, wenn man die gegenseitige Wechselwirkung der beiden Teilchen, wie vorhin erwähnt, nur in erster Näherung berücksichtigt:

$$
\begin{gathered}
\left(K_{1}^{2}+k_{2}^{2}-E\right) g\left(K_{1} k_{2}\right)-\frac{B}{\pi} \int g\left(K_{1} k_{2}\right) \mathrm{d} k_{2}-\int V \cdot\left(K_{1} \mid k_{1}\right) * \mathrm{~d} k_{1}, \\
\left(k_{2}^{2}-B^{2}-E\right) g\left(k_{2}\right)-\frac{B}{\pi} \int g\left(k_{2}\right) \mathrm{d} k_{2}=-\int V \cdot\left(1_{B} \mid k_{1}\right) * \mathrm{~d} k_{1},\left\{E=K_{0}{ }^{2}-B^{2}+i \alpha\right\} .
\end{gathered}
$$

Hierbei ist$$
V\left(k_{1} k_{2}\right)=\frac{A}{2 \pi} \int f_{0}\left(k_{1}+k, k_{2}-k\right) \mathrm{d} k=\frac{A}{\pi} \frac{N \cdot K_{0} \cdot r}{\left(K_{0}-i B\right)\left(r+i B-K_{0}\right)\left[r^{2}-\left(K_{0}+i B\right)^{2}\right]}, r=k_{1}+k_{2} .
$$

Zunächst betrachten wir Gl. (7 b). Wie man aus ihrer Herleitung sieht, beschreibt die Wellenfunktion $g\left(k_{2}\right)$ den Einfang des Stoßteilchens 1 unter Ionisation des gebundenen Teilchens 2 (Austauschstreuung). Um Gl. (7 b) zu lösen, setzt man

$$
\begin{aligned}
& \int g\left(k_{2}\right) \mathrm{d} k_{2}=C \\
& \text { mit } \quad \int V \cdot\left(1_{B} \mid k_{1}\right) * \mathrm{~d} k_{1}=\frac{A}{\pi} \frac{K_{0} B^{2}}{K_{0}-i B} \frac{3 k_{2}+K_{0}+2 i B}{\left(\left(k_{2}+2 i B\right)^{2}-K_{0}^{2}\right)\left(k^{2}-K_{0}-2 i B\right)} \text {. }
\end{aligned}
$$

und erhält damit

Zur Bestimmung von $C$ wird (9) in (8) eingesetzt und man erhält daraus $C=-i A K_{0} / 4\left(K_{0}-i B\right)^{2}$. (10)

Für den asymptotischen Anteil von $g\left(k_{2}\right)\left(k_{2} \rightarrow \pm K_{0}\right)$, der allein für die Bestimmung des Austauschstreuquerschnitts maßgebend ist, ergibt sich damit:

$$
g\left(k_{2}\right)_{\text {Austausch }}=\left\{\begin{array}{l}
-\frac{A K_{0}{ }^{3}}{2 \pi\left(K_{0}^{2}+B^{2}\right)\left(K_{0}-i B\right)} \frac{1}{k_{2}^{2}-K_{0}^{2}-i \alpha}, k_{2} \rightarrow+K_{0} \\
-\frac{i A K_{0}^{2} B}{2 \pi\left(K_{0}{ }^{2}+B^{2}\right)\left(K_{0}-i B\right)} \frac{1}{k_{2}{ }^{2}-K_{0}{ }^{2}-i \alpha}, k_{2} \rightarrow-K_{0} .
\end{array}\right.
$$

Die Wellenfunktion $g\left(K_{1} k_{2}\right)$ in Gl. (7 a) beschreibt für $K_{1}{ }^{2}+k_{2}{ }^{2} \rightarrow E(E>0)$ die Ionisationsstreuung der beiden Teilchen (beide Teilchen sind nach dem Stoß nicht mehr an den Ursprung gebunden). Für $K_{1} \rightarrow \pm K_{0}\left(E=K_{0}^{2}-B^{2} \gtrless 0\right)$ wird durch $g\left(K_{1} k_{2}\right)$ die rein elastische Streuung des Teilchens 1 am ge-

${ }^{6}$ Der Imaginärteil $-i \alpha$ bewirkt, da $\beta$ die Streuwelle der Teilchen 1 (bzw. 2) asymptotisch durch eine auslaufende Welle dargestellt wird, wie es allein physika- bundenen Teilchen 2 und am Ursprung beschrieben. Für die näherungsweise Darstellung der Ionisationsstreuung reicht die Bornsche Entwicklung aus ${ }^{1}$. Daher soll die Ionisationsstreuung nicht weiter betrachtet werden, da es uns hier nach dem am Anfang Gesagten darauf ankommt, die Austausch-

lisch sinnvoll ist ${ }^{1,7}$. Man kann dies leicht durch Umschreibung von $(5 \mathrm{~b})$ in den Ortsraum erkennen.

${ }^{7}$ W. Heisenberg, Z. Physik 120, 519 [1943]. 
streuung zu untersuchen, bei der die Wechselwirkung der Stoßteilchen mit dem Ursprung nicht mehr als klein betrachtet werden darf. Dagegen muß die Wellenfunktion für die elastische Streuung der Teilchen 1 berechnet werden, da in die Be- stimmung des Gesamtstreuquerschnitts Interferenzglieder zwischen elastischer Streuung und Austauschstreuung eingehen, sobald man Probleme betrachtet, bei denen in Analogie zu den Mehrelektronenproblemen die Teilchen ununterscheidbar sind.

Aus Gl. (7a) erhält man ganz analog zu den Verfahren zur Bestimmung von $g\left(k_{2}\right)$

$g\left(K_{1} k_{2}\right)=\left\{-\frac{B}{\pi} \frac{\sqrt{E-K_{1}^{2}}}{\sqrt{E-K_{1}^{2}}-i B} \int \frac{V \cdot\left(K_{1} \mid k_{1}\right)^{*} \mathrm{~d} k_{1}}{K_{1}{ }^{2}+k_{2}^{2}-E} \mathrm{~d} k_{2}-\int V \cdot\left(K_{1} \mid k_{1}\right) * \mathrm{~d} K_{1}\right\} \frac{1}{K_{1}{ }^{2}+k_{2}{ }^{2}-E}$.

Geht man mittels der Transformation $f_{1}^{\prime}\left(k_{1} k_{2}\right)=\int g\left(K_{1} k_{2}\right)\left(K_{1} \mid k_{1}\right) \mathrm{d} k_{1}$ in den Impulsraum über und spaltet für $k_{1} \rightarrow \pm K_{0}$ die Eigenfunktion $N /\left(k_{2}{ }^{2}+B^{2}\right)$ für die Bindung des Teilchens 2 am Ursprung ab, so erhält man für den asymptotischen Anteil der elastischen Streuwelle:

$$
g\left(k_{1}\right)_{\text {Str. }}=\left\{\begin{array}{l}
\frac{-A K_{0}{ }^{3}}{2 \pi\left(K_{0}-i B\right)\left(K_{0}{ }^{2}+B^{2}\right)} \frac{1}{k_{1}{ }^{2}-K_{0}{ }^{2}-i \alpha}, k_{1} \rightarrow+K_{0} \\
-\frac{i A K_{0}{ }^{2} B}{2 \pi\left(K_{0}-i B\right)\left(K_{0}{ }^{2}+B^{2}\right)} \frac{1}{K_{1}{ }^{2}-K_{0}-i \alpha}, k_{1} \rightarrow-K_{0} .
\end{array}\right.
$$

Zur Herleitung von (12b) sei noch bemerkt, daß für den asymptotischen Anteil der elastischen Streuwelle nur das erste Glied in (12a) maßgebend ist, denn allein dieses Glied besitzt wegen des Faktors $\sqrt{E-K_{1}^{2}} /\left(\sqrt{E-K_{1}^{2}}-i B\right)$ lineare Singularitäten für $K \rightarrow \pm K_{0}$.

Betrachtet man in (7a) die Konstante $B$ als kleine Größe und versucht (7a) durch successive Approximation nach Potenzen von $B$ zu lösen, so erhält man für $g\left(K_{1} k_{2}\right)$ statt (12a)

$$
\begin{array}{r}
g\left(K_{1} k_{2}\right)=-\left\{-\frac{B}{\pi}\left[1+\frac{i B}{\sqrt{E-K_{1}}} \ldots+\left(\frac{i B}{\sqrt{E-K_{1}^{2}}}\right)^{n}+\ldots\right] \cdot \int \frac{V \cdot\left(K_{1} \mid k_{1}\right)^{*} \mathrm{~d} k_{1}}{K_{1}{ }^{2}+k_{2}^{2}-E} \mathrm{~d} k_{2}\right. \\
\left.-\int V \cdot\left(K_{1} \mid k_{1}\right) * \mathrm{~d} k_{1}\right\} \frac{1}{{K_{1}{ }^{2}+k_{2}{ }^{2}-E}^{2}} .
\end{array}
$$

Statt des Faktors $\sqrt{E-K_{1}^{2}} /\left(\sqrt{E-K_{1}^{2}}-i B\right)$ tritt jetzt also eine Potenzreihenentwicklung nach Potenzen von $B$ auf, die für $\left|K_{1}\right| \leqq K_{0}$ divergiert. Durch dieses Näherungsverfahren können wir daher nicht die elastische Streuung des Teilchens 1 am Ursprung und am gebundenen Teilchen 2 beschreiben. Im Hinblick auf die nachfolgenden Untersuchungen über das Oppenheimersche Näherungsverfahren ist diese Bemerkung von Wichtigkeit. Der Grund für dieses Versagen besteht darin, daß die Streuwellenfunktion nach den Eigenfunktionen des Teilchens 1 in Wechselwirkung mit dem Ur- sprung entwickelt wurde, anstatt nach den entsprechenden Eigenfunktionen des Teilchens 2. Diese zweite Entwicklung liefert uns das obige Ergebnis über die elastische Streuwelle [Gl. (12b)] ohne Rechnung, da man dazu in (7b) und wegen des in $k_{1}$ und $k_{2}$ symmetrischen $V\left(k_{1} k_{2}\right)$ damit auch in (11) nur den Index 1 mit dem Index 2 zu vertauschen braucht.

Betrachtet man analog zur Elektronenstreuung an Wasserstoffatomen die beiden Teilchen als gleich, so muß man von folgenden Eigenfunktionen 0-ter Näherung (bezüglich $A$ ) ausgehen:

$$
\begin{aligned}
\bar{f}_{0}\left(k_{1} k_{2}\right)=\frac{1}{\sqrt{2}}\left\{\delta\left(k_{1}-K_{0}\right)+\right. & \left.\frac{K_{0}}{K_{0}-i B} \frac{B}{\pi} \frac{1}{k_{1}^{2}-K_{0}{ }^{2}-i \alpha}\right\} \frac{N}{k_{2}^{2}+B^{2}} \\
& \pm \frac{1}{\sqrt{2}}\left\{\delta\left(k_{2}-K_{0}\right)+\frac{K_{0}}{K_{0}-i B} \frac{B}{\pi} \frac{1}{k_{2}{ }^{2}-K_{0}{ }^{2}-i \alpha}\right\} \frac{N}{k_{1}{ }^{2}+B^{2}} .
\end{aligned}
$$


Das +-Zeichen gehört dabei zur symmetrischen und das --Zeichen zur antisymmetrischen Eigenfunktion. Der Faktor 1/ $\sqrt{2}$ bewirkt, daß die Anzahl der einlaufenden Teilchen die gleiche ist wie beim unsymmetrischen Beispiel. Da die Wellenfunktionen zu unserem Problem durch lineare Integralgleichungen beschrieben werden, können durch Überlagerung von Lösungen neue Lösungen dieser Integralgleichungen gewonnen werden. Wendet man diese Methode zur Gewinnung der symmetrischen Lösung an, so erhält man für die elastische und Austausch-Streuwelle zusammen in erster Näherung bezüglich $A$ :

$$
\begin{aligned}
\bar{f} \mathrm{el}=\frac{1}{\sqrt{2}}\left\{\left(\frac{K_{0}}{K_{0}-i B}\right.\right. & \left.\frac{B}{\pi} \frac{1}{k_{1}^{2}-K_{0}^{2}}+2 g\left(k_{1}\right)\right) \frac{N}{k_{2}{ }^{2}+B^{2}} \\
& \left.+\left(\frac{K_{0}}{K_{0}-i B} \frac{B}{\pi} \frac{1}{k_{2}{ }^{2}-K_{0}{ }^{2}}+2 g\left(k_{2}\right)\right) \frac{N}{k_{1}{ }^{2}+B^{2}}\right\} ; k_{1} \text { bzw. } k_{2} \rightarrow \pm K_{0} .
\end{aligned}
$$

Hierbei ist $g\left(k_{1}\right)=g_{\text {str. }}\left(k_{1}\right)=g_{\text {Aust. }}\left(k_{1}\right)$. Das Entsprechende gilt für $g\left(k_{2}\right)$.

Bei der Herleitung der antisymmetrischen Streuwelle stellte sich heraus, daß hier durch die gegenseitige Wechselwirkung der beiden Teilchen keine Streuwelle verursacht wird. Das ist verständlich, da in einem antisymmetrischen Zustand sich die beiden Teilchen nie am selben Ort befinden können und sie daher wegen ihrer $\delta$-funktionsartigen Wechselwirkung nicht miteinander wechselwirken können. Wie man sich leicht überlegen kann, gilt dies auch für die strenge antisymmetrische Lösung.

\section{Oppenheimersche Näherung}

Als Ausgangsgleichung nehmen wir wieder Gl. (3) Wie am Anfang erwähnt wurde, besteht der Unterschied zwischen der Bornschen und Oppenheimerschen Näherung im wesentlichen darin, daß im Gegensatz zur Bornschen Näherung bei der Oppenheimerschen Näherung für die Wellenfunktion 0-ter Näherung ein in den Teilchenkoordinaten symmetrischer (bzw. antisymmetrischer) Ansatz gewählt wird $^{3}$. Für die Wellenfunktion wird daher jetzt folgender Ansatz gemacht:

$$
f\left(k_{1} k_{2}\right)=\bar{f}_{0}\left(k_{1} k_{2}\right)+\bar{f}_{1}\left(k_{1} k_{2}\right)
$$

mit

$$
\begin{aligned}
& \bar{f}_{0}\left(k_{1} k_{2}\right)=f_{0}^{\mathrm{a}}\left(k_{1} k_{2}\right)+f_{0}^{\mathrm{b}}\left(k_{1} k_{2}\right)=\frac{1}{\sqrt{2}} \quad(16 \mathrm{~b}) \\
& \cdot\left\{\delta\left(k_{1}-K_{0}\right) \frac{N}{k_{2}^{2}+B^{2}}+\delta\left(k_{2}-K_{0}\right) \frac{N}{k_{1}{ }^{2}+B^{2}}\right\} .
\end{aligned}
$$

Wie man aus (16 b) sieht, ist in der Wellenfunktion 0 -ter Näherung $f_{0}\left(k_{1} k_{2}\right)$ die Streuung der freien Teilchen am Ursprung noch nicht berücksichtigt. Das läßt sich im allgemeinen Fall auch nicht durchführen. Denn berücksichtigt man z. B. bei der Streuung von Elektronen an Wasserstoffatomen die endliche Masse des Wasserstoffkerns, so findet über diesen Kern bereits eine Kraftübertragung zwischen dem Stoßelektron und dem gebundenen Elektron statt, wenn man in 0-ter Näherung auch die Wechselwirkung zwischen diesen Elektronen vernachlässigt. Das heißt, man hat dann bereits in 0-ter Näherung ein Dreikörperproblem vor sich, das sich nicht streng lösen läßt.

$\bar{f}_{1}\left(k_{1} k_{2}\right)$ wird bei dieser Näherungsmethode ana$\log \mathrm{zu}$ vorhin ebenfalls nach den Eigenfunktionen des verkürzten Hamilton-Operators (3) $(A=0$, $\left.B_{2}=0\right)$ entwickelt. Für die Entwicklungskoeffizienten $g\left(K_{1} k_{2}\right)$ und $g\left(k_{2}\right)$ ergibt sich analog zu den Gleichungen (7) jetzt folgendes noch strenge Gleichungssystem:

$$
\begin{aligned}
& \left(K_{1}{ }^{2}+k_{2}{ }^{2}-E\right) \bar{g}\left(K_{1} k_{2}\right)-\frac{\bar{B}}{\pi} \int \bar{g}\left(K_{1} k_{2}\right) \mathrm{d} k_{2}=\frac{\bar{B}}{\pi} \int f_{0}^{\mathrm{a}}\left(k_{1}{ }^{\prime} k_{2}\right) \mathrm{d} k_{1}{ }^{\prime}\left(K_{1} \mid k_{1}\right) * \mathrm{~d} k_{1} \\
& +\frac{\bar{B}}{\pi} \int f_{0}^{\mathrm{b}}\left(k_{1} k_{2}\right) \mathrm{d} k_{2}\left(K_{1} \mid k_{1}\right) * \mathrm{~d} k_{1}-\frac{A}{2 \pi} \int \bar{f}_{1}\left(k_{1}-k, k_{2}+k\right) \mathrm{d} k_{1}\left(K_{1} \mid k_{1}\right) * \mathrm{~d} k_{1} \\
& -\frac{A}{2 \pi} \int\left(f_{0}^{\mathrm{a}}\left(k_{1}-k, k_{2}+k\right)+f_{0}^{\mathrm{b}}\left(k_{1}-k, k_{2}+k\right)\right) \mathrm{d} k\left(K_{1} \mid k_{1}\right) * \mathrm{~d} k_{1}, \\
& \left(k_{2}{ }^{2}-B^{2}-E\right) \bar{g}\left(k_{2}\right)-\frac{\bar{B}}{\pi} \int \bar{g}\left(k_{2}\right) \mathrm{d} k_{2}=\frac{\bar{B}}{\pi} \int f_{0}{ }^{\mathrm{a}}\left(k_{1}{ }^{\prime} k_{2}\right) \mathrm{d} k_{1}{ }^{\prime}\left(\mathbf{1}_{B} \mid k_{1}\right) * \mathrm{~d} k_{1} \\
& +\frac{\bar{B}}{\pi} \int f_{0}^{\mathrm{b}}\left(k_{1} k_{2}\right) \mathrm{d} k_{2}\left(\mathbf{1}_{B} \mid k_{1}\right) * \mathrm{~d} k_{1}-\frac{A}{2 \pi} \int \bar{f}_{1}\left(k_{1}-k, k_{2}+k\right) \mathrm{d} k\left(\mathbf{l}_{B} \mid k_{1}\right) * \mathrm{~d} k_{1} \\
& -\frac{A}{2 \pi} \int\left(f_{0}^{\mathrm{a}}\left(k_{1}-k, k_{2}+k\right)+f_{0}^{\mathrm{b}}\left(k_{1}-k, k_{2}+k\right)\right) \mathrm{d} k\left(1_{B} \mid k_{1}\right) * \mathrm{~d} k_{1} ; \bar{B}=B ; E=K_{0}{ }^{2}-B^{2}+i \alpha .
\end{aligned}
$$


Die Unterscheidung zwischen $B$ und $\bar{B}$ geschieht wieder nur darum, um die späteren Hinweise auf die Gl. (17) zu erleichtern.

Der wesentliche Unterschied der Gl. (17) gegenüber den Gln. (7) besteht darin, daß in (17) noch die Zusatzglieder

$$
\begin{aligned}
& \frac{B}{\pi} \int\left\{\int f_{0}^{\mathrm{a}}\left(k_{1}{ }^{\prime} k_{2}\right) \mathrm{d} k_{1}{ }^{\prime}\right. \\
& \left.\quad+\int f_{0}^{\mathrm{b}}\left(k_{1} k_{2}{ }^{\prime}\right) \mathrm{d} k_{2}{ }^{\prime}\right\}\left(K_{1} \mid k_{1}\right)^{*} \mathrm{~d} k_{1} \\
& \frac{B}{\pi} \int\left\{\int f_{0}^{\mathrm{a}}\left(k_{1}{ }^{\prime} k_{2}\right) \mathrm{d} k_{1}{ }^{\prime}\right. \\
& \left.\quad+\int f_{0}^{\mathrm{b}}\left(k_{1} k_{2}{ }^{\prime}\right) \mathrm{d} k_{2}{ }^{\prime}\right\}\left(1_{B} \mid k_{1}\right)^{*} \mathrm{~d} k_{1}
\end{aligned}
$$

auftauchen. Das kommt daher, daß in 0-ter Näherung noch nicht die Streuung der Teilchen am Ursprung berücksichtigt wurde.

Um die Oppenheimersche Näherungsmethode besser durchschauen zu können, lösen wir (17) in 2 Schritten. Zuerst wird in (17) $f_{0}{ }^{a}=0$ und nachher $f_{0}{ }^{b}=0$ gesetzt. Wegen der Linearität des Gleichungssystems (17) erhalten wir dann durch Überlagerung der beiden Teillösungen die Lösung für unser Problem. Weiter versuchen wir die Gl. (17) bezüglich der Konstanten $\bar{B}$ in beliebig hoher Näherung zu lösen, da nach vorhin hauptsächlich der Einfluß der Wechselwirkung der beiden Teilchen mit dem Ursprung auf die Lösung interessiert. Die gegenseitige Wechselwirkung der beiden Teilchen wird dagegen wie vorhin wieder nur in erster Näherung berücksichtigt. Auch jetzt wird wieder nur die Austauschstreuung und die elastische Streuung der beiden Teilchen betrachtet.

$$
f_{0}^{\mathrm{a}}=0, f_{0}^{\mathrm{b}}=\frac{1}{\sqrt{2}} \delta\left(k_{2}-K_{0}\right) \frac{N}{k_{1}^{2}+B^{2}} .
$$

Diese beiden Beziehungen bedeuten, daß vor dem Stoß das Teilchen 2 den Impuls $K_{0}$ besitzt und das Teilchen 1 an den Ursprung gebunden ist. Die Glieder

$$
\begin{array}{r}
-\frac{A}{2 \pi} \int \bar{f}_{1}\left(k_{1}-k, k_{2}+k\right) \mathrm{d} k \cdot\left(K_{1} \mid k_{1}\right) * \mathrm{~d} k_{1} \text { und } \\
-\frac{A}{2 \pi} \int \bar{f}_{1}\left(k_{1}-k_{1} k_{2}+k\right) \mathrm{dk} \cdot\left(1_{B} \mid k_{1}\right) * \mathrm{~d} k_{1}
\end{array}
$$

dürfen wir jetzt nicht als Glieder 2. Näherung $=0$ setzen. In ihnen ist jetzt ein Anteil enthalten, der die elastische Streuung der Teilchen 2 am Ursprung (keine Wechselwirkung mit den am Ursprung gebundenen Teilchen 1) beschreibt. Wie vorhin bereits erläutert wurde, kann dieser Anteil meist nicht exakt berechnet werden, sondern er muß im allgemeinen Fall ebenfalls durch ein Störungsverfahren berechnet werden. Für diesen Anteil ergibt sich aus (17) durch successive Approximation folgende Potenzreihenentwicklung nach $B$ :

$$
\begin{aligned}
& f_{1}^{\prime}\left(k_{1} k_{2}\right)=\bar{g}_{\substack{A \\
A=0}}^{\prime}\left(k_{2}\right)\left(1_{B} \mid k_{1}\right) \\
& =\frac{1}{\sqrt{2}} \frac{B}{\pi}\left\{1+\frac{i B}{K_{0}}+\left(\frac{i B}{K_{0}}\right)^{2} \ldots\right\} \frac{1}{k_{2}^{2}-K_{0}{ }^{2}-i \alpha} .
\end{aligned}
$$

Hierbei stellt $1+i B / K_{0}+\left(i B / K_{0}\right)^{2} \ldots$ die Potenzreihenentwicklung von $K_{0} /\left(K_{0}-i B\right)$ nach Potenzen von $B$ dar. Setzt man die Entwicklung (18) in (17 b) ein, so erhält man ebenfalls durch successive Approximation folgende Potenzreihenentwicklung für $g^{\prime}\left(k_{2}\right)$, das die elastische Streuung der Teilchen 2 am Ursprung und an den gebundenen Teilchen 1 beschreibt:

für $k_{2} \rightarrow+K_{0}: \bar{g}_{\text {as }}^{\prime}\left(k_{2}\right)=\frac{B}{\sqrt{2} \pi}\left\{1+\left(1+\frac{i B}{K_{0}} \ldots\right) \frac{B i}{K_{0}}+i A\left[1+\frac{i B}{K_{0}} \ldots\right]\left[\frac{-\left(K_{0}+2 i B\right)}{4 K_{0}\left(K_{0}+i B\right)}\right.\right.$

$$
\left.\left.+\frac{B^{2}}{K_{0}}\left(1+\frac{i B}{K_{0}} \ldots\right) \frac{1}{2 K_{0}\left(K_{0}+i B\right)}-\frac{1}{4 K_{0}} \frac{K_{0}+2 i B}{\left(K_{0}+i B\right)}\right]-\frac{A}{2 B}\right\} \frac{1}{k_{2}^{2}-K_{0}^{2}-i \alpha},
$$

für $k_{2} \rightarrow-K_{0}: \bar{g}_{\text {as }}^{\prime}\left(k_{2}\right)=\frac{B}{\sqrt{2} \pi}\left\{1+\left(1+\frac{i B}{K_{0}} \ldots\right) \frac{B i}{K_{0}}+i A\left[1+\frac{i B}{K_{0}} \ldots\right]\left[\frac{-\left(K_{0}^{2}+2 B^{2}+i B K_{0}\right)}{4 K_{0}\left(K_{0}^{2}+B^{2}\right)}\right.\right.$

$$
\left.\left.+\frac{B^{2}}{K_{0}}\left(1+\frac{i B}{K_{0}} \ldots\right) \frac{1}{2 K_{0}\left(K_{0}+i B\right)}-\frac{1}{4 K_{0}} \frac{K_{0}+2 i B}{\left(K_{0}+i B\right)}\right]-\frac{A B}{2\left(K_{0}{ }^{2}+B^{2}\right)}\right\} \frac{1}{k_{2}{ }^{2}-K_{0}{ }^{2}-i \alpha} .
$$

Wie man aus der Herleitung von (19) und durch Vergleich mit den Gln. (4b) und (12b) erkennt, ist diese Streuung als eine Potenzreihenentwicklung (Bornsche Näherung) nach derjenigen Konstanten
$B$ gegeben, die für die Wechselwirkung der Teilchen 2 mit dem Ursprung maßgebend ist.

Weiter erkennt man aus (19), daß diese Entwicklung für $\left|K_{0}\right|<B$ divergiert. Daher kann man die 
elastische Streuung der Teilchen 2 für Stoßenergien kleiner als die Bindungsenergie der Teilchen am Ursprung durch diese Potenzreihenentwicklung nicht mehr beschreiben. Das gilt allerdings nur für eindimensionale Beispiele. Im eindimensionalen Fall ist das Verhältnis der Intensität der Streuwelle zur Intensität der einfallenden Welle viel größer als im dreidimensionalen Fall (bei gleich starker Kopplung; als Maß für die Stärke der Kopplung sei die Bindungsenergie der Teilchen am Ursprung gewählt), da die Teilchen im eindimensionalen Fall einander nicht ausweichen können. In dreidimensionalen Beispielen konvergiert die Bornsche Näherung auch noch für kleinere Stoßenergien. Allerdings wird auch bei dreidimensionalen Problemen für kleine Stoßenergien $\left(E_{\text {Stoß }} \ll E_{\text {Bind. }}\right)$ die Bornsche Entwicklung divergieren.

Es sollen nun noch ein paar Worte zur Austauschstreuung der Teilchen 2 mit den Teilchen 1 gesagt werden. Diese Streuung wird im wesentlichen durch die Gl. (17a) beschrieben. Aber es zeigt sich ganz analog zu vorhin [s. Gl. (12 c)], daß diesmal die Potenzreihenentwicklung für die Austauschstreuung divergiert. Um eine konvergente Potenzreihenentwicklung (wenigstens für einen bestimmten Bereich der Stoßenergie) zu erhalten, müßte man nach den entsprechenden Eigenfunktionen der Teilchen 2 entwickeln. Wir können daher im Rahmen des üblichen Oppenheimerschen Näherungsverfahrens die Austauschstreuung der Stoßteilchen 2 mit den gebundenen Teilchen 1 nicht beschreiben.

$$
f_{0}^{\mathrm{a}}=\frac{1}{\sqrt{2}} \delta\left(k_{1}-K_{0}\right) \frac{N}{{k_{2}}^{2}+B^{2}}, f_{0}^{\mathrm{b}}=0 .
$$

Diesmal besitzt das Teilchen 1 den Impuls $K_{0}$ vor dem Stoß, und das Teilchen 2 ist an den Ursprung gebunden. Als erstes wird jetzt die Austauschstreuung betrachtet. Dafür ist wieder Gl. (17 b) maßgebend, da bei der Austauschstreuung nach dem Stoß das Teilchen 1 an den Ursprung gebunden ist. Die Glieder

und

$$
\begin{aligned}
& -\frac{A}{2 \pi} \int \bar{f}\left(k_{1}-k, k_{2}+k\right) \mathrm{d} k \cdot\left(K_{1} \mid k_{1}\right) * \mathrm{~d} k_{1} \\
& -\frac{A}{2 \pi} \int \bar{f}_{1}\left(k_{1}-k, k_{2}+k\right) \mathrm{d} k\left(1_{B} \mid k_{1}\right) * \mathrm{~d} k_{1}
\end{aligned}
$$

dürfen analog zu vorhin auch jetzt nicht als Glieder 2. Ordnung vernachlässigt werden, da diesmal in ihnen ein Anteil enthalten ist, der die elastische Streuung der Teilchen 1 am Ursprung (keine Wechselwirkung mit den Teilchen 2) beschreibt. Für seine näherungsweise Berechnung ist die Entwicklung nach den Eigenfunktionen des Teilchens 1 in Wechselwirkung mit dem Ursprung nach vorhin [s. z. B. Gl. (12c)] nicht geeignet, sondern man muß ihn nach den entsprechenden Eigenfunktionen des Teilchens 2 entwickeln, um die Streuung des Teilchens 1 am Ursprung als Störung auffassen zu können. Das heißt, man muß diesen Anteil mit Hilfe der Bornschen Näherung berechnen. Analog zu Gl. (18) ergibt sich für diesen Anteil:

$$
\begin{aligned}
\bar{f}_{1}^{\prime \prime} & \left(k_{1} k_{2}\right)=\bar{g}^{\prime \prime}\left(k_{1}\right)\left(1_{B} \mid k_{2}\right) \\
= & \frac{B}{\sqrt{2} \pi}\left\{1+\frac{i B}{K_{0}} \ldots\right\} \frac{1}{{k_{1}}^{2}-K_{0}^{2}-i \alpha} .
\end{aligned}
$$

Geht man mit (20) in (17b) ein, so erhält man für die Austauschstreuung der Stoßteilchen 1 mit den gebundenen Teilchen 2 analog zu Gl. (19):

$$
\begin{aligned}
\text { für } k_{2} \rightarrow+K_{0}: \bar{g}_{\text {as }}^{\prime \prime}\left(k_{1}\right)= & \frac{B}{\sqrt{2} \pi}\left\{\frac{2 B^{2}}{K_{0}{ }^{2}+B^{2}}\left[1-\frac{K_{0}-i B}{K_{0}}\left(1+\frac{i B}{K_{0}} \ldots\right)\right]\right. \\
& +i A\left[1+\frac{i B}{K_{0}} \ldots\right]\left[\frac{-\left(K_{0}+2 i B\right)}{4 K_{0}\left(K_{0}+i B\right)}+\frac{B^{2}}{K_{0}}\left(1+\frac{i B}{K_{0}} \ldots\right) \frac{1}{2 K_{0}\left(K_{0}+i B\right)}\right. \\
& \left.\left.-\frac{\left(K_{0}+2 i B\right)}{4 K_{0}\left(K_{0}+i B\right)}\right]-\frac{A}{2 \pi}\right\} \frac{1}{k_{2}{ }^{2}-K_{0}{ }^{2}-i \alpha}, \\
\text { für } k_{2} \rightarrow-K_{0}: \bar{g}_{\text {as }}^{\prime \prime}\left(k_{1}\right)= & \frac{B}{\sqrt{2} \pi}\left\{\frac{2 B^{2}}{K_{0}{ }^{2}+B^{2}}\left[1-\frac{K_{0}-i B}{K_{0}}\left(1+\frac{i B}{K_{0}} \ldots\right)\right]\right. \\
& +i A\left[1+\frac{i B}{K_{0}} \ldots\right]\left[\frac{-\left(K_{0}{ }^{2}+2 B^{2}+i B K_{0}\right)}{4 K_{0}\left(K_{0}{ }^{2}+B^{2}\right)}+\frac{B^{2}}{K_{0}}\left(1+\frac{i B}{K_{0}} \ldots\right) \frac{1}{2 K_{0}\left(K_{0}+i B\right)}\right. \\
& \left.\left.-\frac{K_{0}+2 i B}{4 K_{0}\left(K_{0}+i B\right)}\right]-\frac{A B}{2\left(K_{0}{ }^{2}+B^{2}\right)}\right\} \frac{1}{k_{2}{ }^{2}-K_{0}{ }^{2}-i \alpha} .
\end{aligned}
$$


Wie man durch Vergleich mit Gl. (11) sieht, ist in (21) jetzt die Austauschstreuung der Stoßteilchen 1 mit den gebundenen Teilchen 2 als eine Potenzreihenentwicklung nach der Konstanten $B$ gegeben. Weiter erkennt man aus (21), daß auch diese Entwicklung für $\left|K_{0}\right|<B$ divergiert. Es gilt daher für diese Austauschstreuung bezüglich der Konvergenz des Verfahrens dasselbe, was vorhin über die elastische Streuung der Teilchen 2 gesagt wurde (auch im Hinblick auf die Übertragung auf dreidimensionale Probleme). Wie man weiter sieht, enthält (21) ein Glied ohne die Kopplungskonstante $A$ als Faktor. Ist dieses Glied von 0 verschieden, so bedeutet das, daß auch ohne Wechselwirkung zwischen den Teilchen 1 und 2 bereits eine Austauschstreuung stattfindet. Das ist natürlich physikalisch unsinnig. Berücksichtigt man in diesem Glied die Potenzreihe $\left(1+i B / K_{0} \ldots\right)$ streng, so sieht man, daß es gleich 0 wird, wie es physikalisch auch sein muß. Berücksichtigt man diese Potenzreihe dagegen nur näherungsweise (z. B. nur in der 1. Näherung), wie es innerhalb der Oppenheimerschen Näherungsmethode getan wird, so verschwindet dieses nicht. Beim Übergang zu dreidimensionalen Beispielen ändert sich daran nichts Wesentliches, da dieses Glied dadurch hervorgerufen wird, daß die Wellenfunktion $f_{0}{ }^{a}$ keine Eigenfunktion des verkürzten Hamilton-Operators (3) ist. Es ist im wesentlichen dafür verantwortlich, daß die Oppenheimersche Näherung für kleine Energien viel zu große Wirkungsquerschnitte liefert ${ }^{3}$. Wir kommen darauf anschließend noch einmal zu sprechen.

Betrachtet man nun die elastische Streuung der Teilchen 1 am Ursprung und an den Teilchen 2, so ist für die Beschreibung dieser Streuung jetzt Gl. (17 a) maßgebend. Aus den bereits früher erläuterten Gründen (s. S. 285 ff.) divergiert die aus ihr zu gewinnende Potenzreihenentwicklung nach dem Kopplungsparameter $\bar{B}$ für die elastische Streuung.

Aus all diesen Betrachtungen ist ersichtlich, daß die Oppenheimersche Näherungsmethode die Ununterscheidbarkeit gleicher Teilchen nicht richtig berücksichtigen kann (s. z. B. ${ }^{3}$ ), da sie für das eine Teilchen (Teilchen 2) nur die elastische Streuung näherungsweise wiedergibt und für das andere Teilchen (Teilchen 1) nur die Austauschstreuung näherungsweise liefert. Um die Symmetrie der Teilchen zu berücksichtigen, muß man daher zur Wellenfunktion die in den Koordinaten 1 und 2 vertauschte Wellenfunktion hinzuaddieren.

Um den Einfluß des vorhin erwähnten Gliedes, das nur dadurch auftritt, daß das Näherungsverfahren bei einer bestimmten Näherung abgebrochen wird, abschätzen zu können, berechnen wir aus der symmetrisierten Lösung von (17 b) den zugehörigen Wirkungsquerschnitt $Q_{\mathrm{I}}$ für elastische Streuung + Austauschstreuung in erster Näherung bezüglich der Konstanten $A$ und $\bar{B}$ und vergleichen ihn mit dem Wirkungsquerschnitt, in $\operatorname{dem} A$ in erster Näherung, dagegen die Kopplungskonstante $B$ in beliebig hoher Näherung berücksichtigt ist. In dem beigefügten Diagramm ist das Verhältnis von $Q_{\mathrm{I}}: Q$ für verschiedene Stoßenergien $K_{0}{ }^{2}$ aufgezeichnet (Kurve I). Die Kurve II stellt das Verhältnis $Q_{\mathrm{II}}: Q$ dar, wobei $Q_{\mathrm{II}}$ aus $Q_{\mathrm{I}}$ hervorgeht, wenn zur Berechnung von $Q_{\mathrm{I}}$ das Glied

$$
\frac{2 B^{2}}{K_{0}^{2}+B^{2}}\left[1-\frac{K_{0}-i B}{K_{0}}(\ldots)\right]
$$

in (21), dessen Auftreten nach oben allein durch das Näherungsverfahren bedingt ist und das bei Aufsummierung über alle Näherungen verschwindet, weggelassen wird.

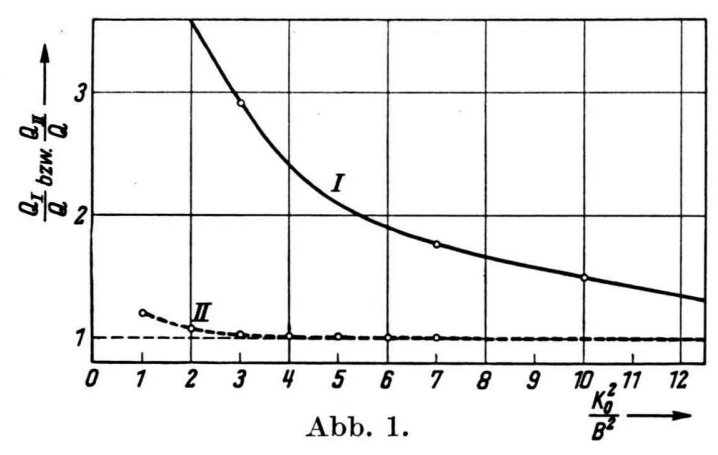

Für diese Wirkungsquerschnitte erhält man folgende Beziehungen:

$$
\begin{aligned}
Q= & \frac{2 B}{K_{0}{ }^{2}+B^{2}}\left\{B-\frac{A K_{0}{ }^{2}}{K_{0}{ }^{2}+B^{2}}\right\} \\
Q_{\mathrm{I}}= & \frac{2 B}{K_{0}{ }^{2}} \frac{K_{0}{ }^{2}+3 B^{2}}{\left(K_{0}{ }^{2}+B^{2}\right)^{2}} \\
& \cdot\left\{B\left(K_{0}^{2}+3 B^{2}\right)-A\left(K_{0}^{2}+2 B^{2}\right)\right\}, \\
Q_{\mathrm{II}}= & \frac{2 B}{K_{0}{ }^{2}}\left\{B-A \frac{K_{0}{ }^{2}+2 B^{2}}{K_{0}{ }^{2}+B^{2}}\right\} .
\end{aligned}
$$

Für hohe Energien $K_{0}{ }^{2}$ streben sämtliche Wirkungsquerschnitte nach demselben Grenzwert

$$
Q_{\text {Grenz. }}=\frac{2 B}{K_{0}^{2}}\{B-A\} .
$$


Wie man aus den beiden Diagrammen abliest, wird der Wirkungsquerschnitt $Q_{\mathrm{I}}$ gegenüber $Q$ für kleine Energien viel zu groß, während $Q_{\mathrm{II}}$ auch für kleine Energien noch relativ gut mit $Q$ übereinstimmt.

Will man mittels der Gl. (17) noch die höheren Näherungen derWellenfunktion bezüglich der Kopplungskonstanten $A$ berechnen, so ändert sich an den eben dargestellten Überlegungen zur Oppenheimerschen Näherung nichts Prinzipielles. Es werden nur die Rechnungen sehr kompliziert. Daher soll auf die Berechnung dieser höheren Näherungen hier verzichtet werden.

\section{Zusammenfassung}

Die Untersuchung der Born-Oppenheimerschen Näherungsmethode an Hand des obigen einfachen Beispiels hat folgendes ergeben:

1. Da diese Näherungsmethode ununterscheidbare Teilchen nicht in gleicher Weise berücksichtigt (s. S. 288 ff.), sind die mit ihrer Hilfe gewonnenen Streuwellenfunktionen auch nicht symmetrisch (bzw. antisymmetrisch) in den Teilchenkoordinaten gleicher Teilchen, obwohl die Wellenfunktion 0-ter Näherung in diesen Koordinaten symmetrisch (bzw. antisymmetrisch) angesetzt wurde. So wird in dem obigen Beispiel für das eine Teilchen nur die elasti- sche Streuung und für das andere Teilchen nur die Austauschstreuung näherungsweise beschrieben. Es muß daher die Streuwellenfunktion nachträglich symmetrisch (bzw. antisymmetrisch) ergänzt werden.

2. Für die Untersuchung dieses Näherungsverfahrens war es praktisch, die verschiedenen Wechselwirkungen der Teilchen einzeln für sich zu betrachten. Dadurch war es möglich, das Auftauchen von Gliedern zu erkennen, die nur auftreten, weil das Näherungsverfahren bei einer bestimmten Näherung abgebrochen wird und die daher physikalisch nicht sinnvoll sind (s. S. $291 \mathrm{ff}$.). Um zu vermeiden, daß die Oppenheimersche Näherung für kleine Energien viel zu großeWirkungsquerschnitte liefert, muß man diese Glieder in der angenäherten Wellenfunktion weglassen. Ganz analog dazu muß man auch bei dreidimensionalen Beispielen vorgehen, um solche Glieder von den physikalisch sinnvollen Gliedern abzutrennen.

3. Wie die Bornsche Näherung divergiert auch die Oppenheimersche Näherung für kleine Energien.

Diese Arbeit wurde im Rahmen eines Forschungsprogramms des Instituts für Mathematik und Mechanik der New York University durchgeführt unter finanzieller Unterstützung des ,G eophysics R esearch Directorate of the Air Force Cambridge Research Center". 